Tropical Journal of Pharmaceutical Research January 2019; 18 (1): 157-165

ISSN: $1596-5996$ (print); 1596-9827 (electronic) (c) Pharmacotherapy Group, Faculty of Pharmacy, University of Benin, Benin City, 300001 Nigeria.

\title{
Human leukocyte antigen I and levels of expression of antigen-presenting element proteins plays a role in triple negative breast cancer
}

\author{
Zhu Xianglu', Gulizhareye Aikula', Mukedaisi Baiketiyaer², Zhang Yujing ${ }^{2}$, \\ Munire Mushajiang ${ }^{1}$ \\ ${ }^{1}$ Breast Radiology Department of Affiliated Cancer Hospital of Xinjiang Medical University, 830001, ${ }^{2}$ Radiology Department of \\ Cancer Center of Sun Yat-Sin University, Urumqi City 510000, China
}

${ }^{*}$ For correspondence: Email: pz1170@163.com

Sent for review: 24 October 2018

Revised accepted: 28 December 2018

\begin{abstract}
Purpose: To determine the protein expression levels of leukocyte antigen I and antigen-presenting element (APM) genes, and to study their relationship with triple negative breast cancer (TNBC) in patients from Uyghur and Han, China.

Methods: Immunohistochemistry was used to determine the expression levels of 10 proteins (HLA-A (Human Leukocyte Antigens-A), HLA-B, HLA-C, TAP1 (Transporter associated with Antigen Processing-1), TAP2, calreticulin, calnexin, ERp57 (Endoplasmic reticulum resident protein 57), ERAP1 (Type 1 tumor necrosis factor receptor shedding aminopeptidase regulator) and tapasin) in TNBC and non-TNBC tissue specimens, and 26 benign lesions (fibrous adenoma) specimens from 120 Uygur and Han women.

Results: Immunohistochemical analysis showed that the positive expressions of HLA-A, HLA-B, TAP2, Erp57, ERAP1, calnexin and calreticulin in breast cancer tissues were significantly lower than those in breast fibroma tissues $(p<0.05)$. Among the 86 TNBC patients, there were 35 cases of tapasin- $(40.70$ $\%), 26$ cases of tapasin+ (30.30\%), and 25 cases of tapasin++ (29.10\%). Among the 34 non-TNBC patients, there were 25 cases of tapsin- (73.50\%), 7 cases of tapasin+ $(20.60 \%)$ and 2 cases of tapasin $++(5.9 \%)$. The positive expression of tapasin in TNBC patients was significantly higher than that in non-TNBC patients $(p<0.05)$.

Conclusion: Down-regulation of transcriptional expression or loss of protein expression of HLA-I and APM genes is closely related to the progression of breast cancer, and is therefore, a potential molecular marker for screening tumors.
\end{abstract}

Keywords: Triple negative breast cancer, Antigen presenting element, Human leukocyte antigen (HLA)

This is an Open Access article that uses a funding model which does not charge readers or their institutions for access and distributed under the terms of the Creative Commons Attribution License (http://creativecommons.org/licenses/by/4.0) and the Budapest Open Access Initiative (http://www.budapestopenaccessinitiative.org/read), which permit unrestricted use, distribution, and reproduction in any medium, provided the original work is properly credited.

Tropical Journal of Pharmaceutical Research is indexed by Science Citation Index (SciSearch), Scopus, International Pharmaceutical Abstract, Chemical Abstracts, Embase, Index Copernicus, EBSCO, African Index Medicus, JournalSeek, Journal Citation Reports/Science Edition, Directory of Open Access Journals (DOAJ), African Journal Online, Bioline International, Open-J-Gate and Pharmacy Abstracts

\section{INTRODUCTION}

Triple negative breast cancer, a subtype of breast cancer, refers to breast cancer that does not express PR, ER and HER-2. Generally,
TNBC occurs in young females. Tumor cells usually generate tumor-related antigens, and the immune system can recognize, monitor and eliminate these antigens [1]. When the antigen presenting function is defective or fails, and when tumor cells get the ability to overwhelm the 
immune system during the interaction between immune system and tumor cells, the tumor cells can survive and develop [2].

As an important surface marker in cells, HLA is closely related to malignant cancer and it plays an essential role in immune regulation and immune response. The structure and function of APM are very important in the process of endogenous antigen peptide presentation in vivo. When its structure and function are impaired, tumor cells can get rid of the surveillance by the immune system [3]. The present study was carried out to determine the protein expression levels of HLA-I and APM in cancer tissue samples from Uyghur and Han TNBC patients, and to investigate the relationship between these proteins and TNBC.

\section{EXPERIMENTAL}

\section{Reagents}

Immunohistochemistry SP kit, DAB developer, EDTA antigen repair solution and PBS buffer solution were purchased from Shanghai Haling Biological Co., Ltd (Shanghai, China). Bromophenol blue was product of Sigma (US); paraffin tissue DNA extraction kit was purchased from Qiagen, Germany; DEPC Amresco (America), mouse anti-human HLA-A, mouse anti-human HLA-B, mouse anti-human HLA-C, rabbit anti-human TAP1, rabbit anti-human TAP2, and rabbit anti-human calreticulin antibodies were products of Beijing Labest Biological Co., Ltd (Beijing). Agar agar was obtained from Shanghai Sangon Biotech (Shangai), while rabbit anti-human ERAP1 and rabbit anti-human ERP57 antibodies were from Zhongshan Jinqiao Biological Company (Beijing). Rabbit anti-human calnexin antibody and mouse anti-human tapasin antibody were purchased from Beijing Labest Biological Co., Ltd (Beijing). Absolute ethanol and xylene were products of Beijing Biochemical Company (Beijing).

\section{Collection of clinical samples}

Tumor tissue samples were collected from 120 females with breast cancer and 26 patients with breast fibroadenoma from the Affiliated Cancer Hospital of the Third Clinical Medical College of Xinjiang Medical University. This study obtained approval from the ethnical committee of the same hospital. The age, medical history, ethnic background, marital status, menstrual history, and family history of the patients were obtained and recorded; the informed consent of the patients were obtained.
This research was approved by the Ethical Committee of Breast Radiology Department of Affiliated Cancer Hospital of Xinjiang Medical University, 789 East Suzhou Rd, Urumchi, Xinjiang Uyghur Autonomous Region (approval no. 20189368), and carried out according to the guidelines of Declaration of Helsinki promulgated in 1964 as amended in 1996 [4].

\section{Inclusion and exclusion criteria}

The patients who were included were those aged from 30 to 70 years who were not on chemotherapy or radiotherapy, and had no other cancers; patients confirmed with primary breast cancer through pathology; patients without disorders in other vital organs such as heart and lung dysfunction as well as liver and kidney failure, and those with complete medical records. Patients in the following categories were excluded from the study: patients unfit for operation due to organic lesions in vital organs, breast cancer patients who received treatment prior to the operation, advanced breast cancer patients with distal metastases, and patients with incomplete medical records.

\section{Method of sample collection}

Tumor tissue samples (120) were taken from Uyghur women and Han women with TNBC and non-TNBC by operation, and 26 samples of benign lesion tissues were taken from patients with fibroadenoma, one of which was used for pathological immunohistochemical identification and the other was immediately frozen in liquid nitrogen and stored in an ultra-low temperature freezer at $-80^{\circ} \mathrm{C}$.

\section{Paraffin embedding of tissues}

Fibroadenoma or breast cancer tissues were embedded in paraffin and cut into slices using conventional methods. The tissues were fixed in $10 \%$ formaldehyde for about $4-6 \mathrm{~h}$. The fixed tissues were dehydrated in ethanol gradient for about $2 \mathrm{~h}$, and cleared in xylene for about $1 \mathrm{~h}$. The transparent tissues were then embedded in paraffin, solidified and trimmed, followed by slicing into 4 sheets of 3 to $4 \mu \mathrm{m}$ thickness. The slices were kept in an oven at $60^{\circ} \mathrm{C}$ overnight.

\section{Preparation of tissue slides and immunohistochemical assays}

Glass slides were rinsed and soaked in tap water overnight. The glass slides were then soaked in potassium dichromate overnight, washed in tap water, and then re-rinsed in tap water and dried. The slides were placed in APES for $30 \mathrm{~s}$ and 
then taken out, followed by washing in distilled water and heating at $60^{\circ} \mathrm{C}$ overnight. The tissues were cut into 12 slices continuously with a blade and kept in an oven at $60{ }^{\circ} \mathrm{C}$ overnight. Thereafter, the slices were placed in incubator for $1 \mathrm{~h}$, and dewaxed with xylene. They were then dehydrated in ethanol gradient and soaked in distilled water for $1 \mathrm{~min}$, followed by antigen retrieval. In this process, the slices in Tris-EDTA solution ( $\mathrm{pH}$ 9.0) were heated in a microwave oven for about $20 \mathrm{~min}$, and then cooled for $30-$ $40 \mathrm{~min}$, followed by washing with PBS (pH 7.6). Then, the slices were blocked in $3 \%$ hydrogen peroxide solution for about $20 \mathrm{~min}$.

This was followed by antigen-antibody binding: $30 \mu \mathrm{l}$ of diluted primary antibody (HLA-A, HLA-B, HLA-C, TAP1, TAP2, calreticulin, calnexin, ERp57, ERAP1 and tapasin) solutions were added dropwise and incubated overnight at $4{ }^{\circ} \mathrm{C}$. Thereafter, diluted biotin-labeled secondary antibody solution was added dropwise and incubated at $37^{\circ} \mathrm{C}$ for $1 \mathrm{~h}$, followed by addition of DAB chromogenic reagent. Hematoxylin was used to counterstain the nuclei for $25-60 \mathrm{sec}$, and the slices were rinsed in tap water, placed in hot water at $60-70{ }^{\circ} \mathrm{C}$ for them to turn blue, and dehydrated with gradient ethanol. They were then cleared with xylene and sealed using neutral gum. Positive and blank controls were set up simultaneously.

\section{Staining techniques}

The staining results were determined by doubleblind microscopy, and the number of positive cells and the degree of staining were used as criteria. The criterion for APM-positive cells was appearance of brownish-yellow or yellowish stain on the cytoplasm or nucleus. For HLA-I positive cells, the cytoplasm or cell membrane were stained yellow or brownish yellow. The number of positive cells and the intensity of staining were scored by reference to literature. The score was based on the proportion of positive cells in epithelial cells viz: 0 score for $<5 \%, 1$ point for proportions $\geq 5$ but $\leq 25 \%, 2$ points for proportions $\geq 26$ but $\leq 75 \%$, and 3 points for proportions $>75 \%$. Staining intensity was scored viz: 0 point for dim staining or absence of cell staining, 1 point for yellowish staining, 2 points for brownish-yellow staining, and 3 points for dark brown staining. The comprehensive score (S) was obtained as in Eq 1.

$\mathrm{S}=(\mathrm{C}+\mathrm{T}) / 2$

where $C$ and $T$ are cell staining and staining intensity scores, respectively.
The criteria used for comprehensive judgment/assessment were: complete absence for comprehensive scores < 0.5 points; partially missing for comprehensive scores in the range of 0.5 to 1.5 points; and normal expression for comprehensive scores $>1.6$ points.

\section{Statistical analysis}

Data for gene transcription expression levels are expressed as mean $\pm S D$, and were compared with $t$-test. Wilcoxon test was used for comparison between two groups. All data were analyzed using SPSS ver. 20.0 software. Values of $p$ were bilateral, and when $p<0.05$, the difference was considered statistically significant.

\section{RESULTS}

\section{Histomorphological features}

Immunohistochemical stains of HLA-I proteins (HLA-A, HLA-B and HLA-C), and APM proteins (TAP1, TAP2, calnexin, calreticulin, tapasin, Erp57 and ERAP1) were observed under the microscope (Figure 1). All of them appeared as brownish-yellow particles, and the protein molecules of HLA-A. The HLA-B and HLA-C protein were mainly located in the cell membrane (Figure 1).
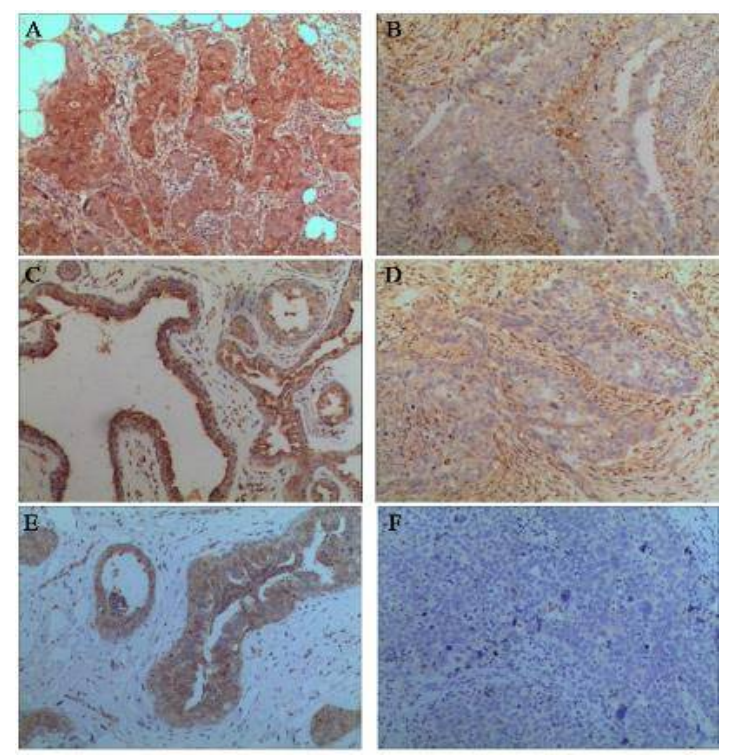

Figure 1: Expression of HLA-A, HLA-B and HIA-C. Note: A, strong expression of HLA-A in benign breast tissues (200x); B, expression loss of HLA-A in breast cancer tissues $(200 \times)$; C, strong expression of HLA-B in benign breast tissues (200x); D, expression loss of HLA-B in breast cancer tissues (200x); E, strong expression of HLA-C in benign breast tissues (200x); $F$, expression loss of HLA-C in breast cancer tissues $(200 \times)$ 
The proteins, Erp57, TAP1, TAP2, calnexin, calreticulin, and tapasin were mainly expressed in the cytoplasm. Proteins TAP1 and TAP2 were partially or negatively expressed in most of the breast cancer tissues, but strongly expressed in breast fibroadenoma tissues (Figure 2).

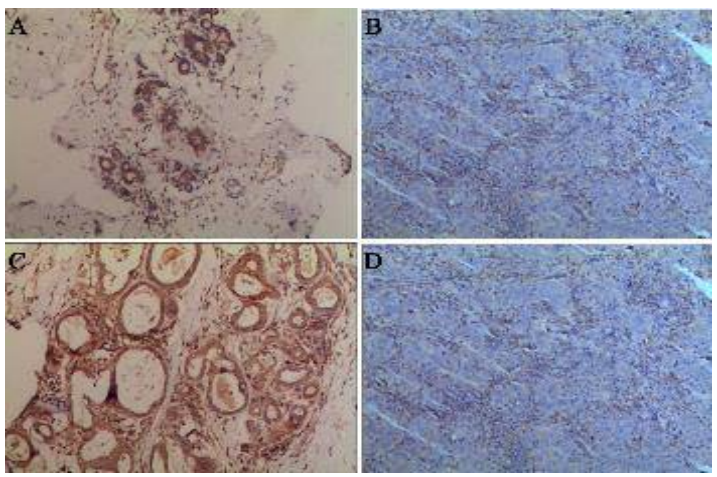

Figure 2: Expression of TAP1 and TAP2. Note: A, strong expression of TAP1 in benign breast tissues (200x); B, expression loss of TAP1 in breast cancer tissues (200x); C, strong expression of TAP2 in benign breast tissues $(200 x)$; D, expression loss of TAP2 in breast cancer tissues $(200 \times)$

Calnexin and calreticulin proteins were expressed mainly in breast cancer tissues with partial expression loss but without complete loss of expression, and they were strongly expressed in breast fibroadenoma tissues. Positive expression of tapasin protein was localized in the cytoplasm, and was mostly distributed as plaques in the tumor cells of breast cancer tissues, diffusely distributed in extracellular matrix, and strongly expressed in brownish yellow in breast fibroadenoma tissues (Figure 3). In breast fibroadenoma, the positive expressions of Erp57 and ERAP1 showed brownish-yellow or chocolate-brown granules under the microscope. Their expressions were down-regulated in cancer tissues and cells (Figure 4).

\section{Expression levels of APM member proteins in benign and breast cancer tissues}

The positive expressions of HLA-A, HLA-B, TAP2, calnexin, calreticulin, Erp57 and ERAP1 were much lower in breast cancer tissues than those in breast fibroadenoma $(p<0.05$, Table 1$)$. There were no significant differences in the expressions of HLA-C, TAP1 and tapasin between benign and breast cancer tissues $(p>$ 0.05 , Table 2).

\section{Expression levels of APM member proteins in Uyghur and Han nationalities}

There were no statistical differences in the expression levels of the HLA-I proteins HLA-A,
HLA-B and HLA-C between Uyghur and Han breast cancer patients. Similarly, no statistical differences were seen in the expression levels of members of the APM family such as TAP1, TAP2, calreticulin, tapasin, Erp57 and ERAP1, between the two groups of breast cancer patients $(p>0.05)$. These results are shown in Table 3 .

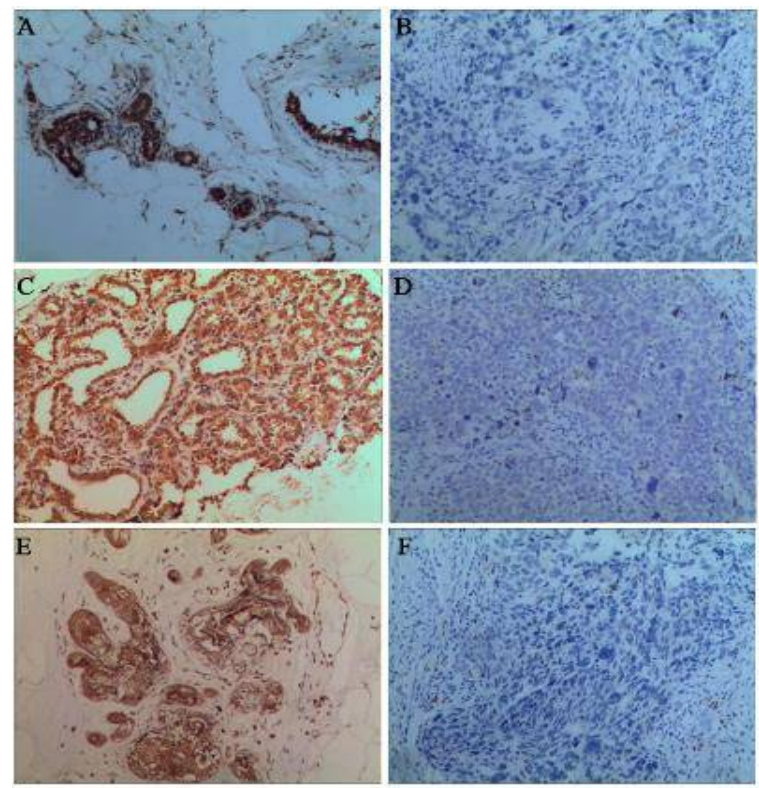

Figure 3: Expression of calnexin, calreticulin, and tapasin. Note: A, strong expression of calnexin benign breast tissues (200x); B, expression loss of calnexin in breast cancer tissues (200x); C, strong expression of calreticulin in benign breast tissues (200x); D, expression loss of calreticulin in breast cancer tissues $(200 \times)$; E, strong expression of tapasin in benign breast tissues $(200 \times)$; F, expression loss of tapasin in breast cancer tissues $(200 \times)$

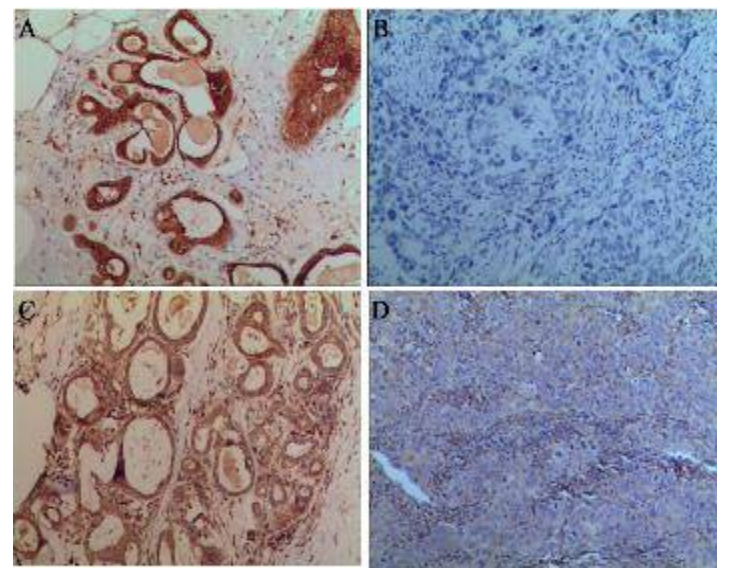

Figure 4: Expression of Erp57 and ERAP1. Note: A, strong expression of Erp57 in benign breast tissues (200x); B, expression loss of Erp57 in breast cancer tissues (200x); C, strong expression of ERAP1 in benign breast tissues $(200 \times)$; D, expression loss of ERAP1 in breast cancer tissues (200x)

Trop J Pharm Res, January 2019; 18(1): 160 
Table 1: Expression levels of HLA-A, HLA-B, TAP2, calnexin, calreticulin, Erp57 and ERAP1 in benign and breast cancer tissues

\begin{tabular}{|c|c|c|c|c|c|}
\hline \multirow[t]{2}{*}{ Index } & \multicolumn{2}{|c|}{$\begin{array}{l}\text { Breast cancer tissues } \\
\qquad(n=120)\end{array}$} & \multicolumn{2}{|c|}{$\begin{array}{l}\text { Breast fibroadenoma } \\
(n=26)\end{array}$} & \multirow[t]{2}{*}{$P$-value } \\
\hline & $N$ & $\%$ & $n$ & $\%$ & \\
\hline HLA-A & & & & & 0.000 \\
\hline- & 66 & 55.00 & 3 & 11.50 & \\
\hline+ & 40 & 33.30 & 8 & 30.80 & \\
\hline++ & 14 & 11.70 & 15 & 57.70 & \\
\hline HLA-B & & & & & 0.000 \\
\hline- & 111 & 92.50 & 17 & 65.40 & \\
\hline+ & 8 & 6.70 & 7 & 26.90 & \\
\hline++ & 1 & 0.80 & 2 & 7.70 & \\
\hline \multicolumn{6}{|l|}{ TAP2 } \\
\hline- & 56 & 46.67 & 4 & 15.38 & \\
\hline+ & 50 & 41.67 & 16 & 61.54 & \\
\hline++ & 14 & 11.67 & 6 & 23.08 & \\
\hline Calnexin & & & & & 0.043 \\
\hline- & 64 & 53.33 & 12 & 46.15 & \\
\hline+ & 42 & 35.00 & 6 & 23.08 & \\
\hline++ & 14 & 11.67 & 8 & 30.77 & \\
\hline \multicolumn{6}{|c|}{ Calreticulin } \\
\hline- & 75 & 62.50 & 19 & 73.08 & 0.010 \\
\hline+ & 29 & 24.17 & 0 & 0.00 & \\
\hline++ & 16 & 13.33 & 7 & 26.92 & \\
\hline ERAP1 & & & & & 0.000 \\
\hline- & 66 & 55.00 & 4 & 15.38 & \\
\hline+ & 34 & 28.33 & 10 & 38.46 & \\
\hline++ & 20 & 16.67 & 12 & 46.15 & \\
\hline Erp57 & & & & & 0.000 \\
\hline- & 50 & 41.67 & 0 & 0.00 & \\
\hline+ & 34 & 28.33 & 12 & 46.15 & \\
\hline++ & 36 & 30.00 & 14 & 53.85 & \\
\hline
\end{tabular}

Table 2: Expression levels of HLA-C, TAP1 and tapasin in benign and breast cancer tissues

\begin{tabular}{cccccc}
\hline \multirow{2}{*}{ Index } & \multicolumn{2}{c}{ Benign cancer tissues } & \multicolumn{2}{c}{ Breast cancer tissues } & \multirow{2}{*}{$\boldsymbol{P}$-value } \\
\cline { 2 - 5 } & $\boldsymbol{N}$ & $\%$ & $\boldsymbol{n}$ & $\%$ & 0.675 \\
HLA-C & 114 & 95.00 & 24 & 92.30 & \\
- & 5 & 4.20 & 2 & 7.70 & 0.072 \\
+ & 1 & 0.80 & 0 & 0.00 & \\
TAP1 & & & & 96.15 & 0.011 \\
- & 117 & 97.50 & 25 & 0.00 & 0.485 \\
+ & 3 & 2.50 & 0 & 3.85 & \\
++ & 0 & 0.00 & 1 & 46.15 & \\
Tapasin & & & & 38.46 & \\
- & 60 & 50.00 & 12 & 15.38 & \\
+ & 33 & 27.50 & 4 & & \\
++ & 27 & 22.50 & & &
\end{tabular}

\section{Expression levels of APM member proteins in TNBC patients and non-TNBC patients}

The results of comparisons of the expression levels of HLA-I member proteins (HLA-A, HLA-B and JLA-C), and APM member proteins (TAP1, TAP2, calnexin, calreticulin, Erp57 and ERAP1) between TNBC patients and non-TNBC patients are shown in Table 4.

\section{DISCUSSION}

Breast cancer is a malignant tumor with high heterogeneity: the various types differ in clinical and pathological features, biological behavior, immune-phenotyping, histomorphology and response to therapy. Gene expression profiles are closely associated with diseases, making cancer molecular classification and individual treatment a reality, 
Table 3: Expression levels of APM member proteins in Uyghur and Han nationalities

\begin{tabular}{|c|c|c|c|c|c|}
\hline \multirow[t]{2}{*}{ Index } & \multicolumn{2}{|c|}{$\begin{array}{l}\text { Uyghur } \\
(n=63)\end{array}$} & \multicolumn{2}{|c|}{$\begin{array}{c}\text { Han } \\
(n=57)\end{array}$} & \multirow[t]{2}{*}{$P$-value } \\
\hline & $N$ & $2 \%$ & $n$ & $\%$ & \\
\hline HLA-A & & & & & 0.740 \\
\hline- & 33 & 52.40 & 33 & 57.90 & \\
\hline+ & 23 & 36.50 & 17 & 29.80 & \\
\hline++ & 7 & 11.10 & 7 & 12.30 & \\
\hline HLA-B & & & & & 0.564 \\
\hline- & 59 & 93.70 & 52 & 91.20 & \\
\hline+ & 4 & 6.30 & 4 & 7.00 & \\
\hline++ & 0 & 0.00 & 1 & 1.80 & \\
\hline HLA-C & & & & & 0.266 \\
\hline - & 59 & 93.70 & 55 & 96.50 & \\
\hline+ & 4 & 6.30 & 1 & 1.80 & \\
\hline++ & 0 & 0.00 & 1 & 1.80 & \\
\hline TAP1 & & & & & 0.619 \\
\hline- & 61 & 96.80 & 56 & 98.20 & \\
\hline+ & 2 & 3.20 & 1 & 1.80 & \\
\hline TAP2 & & & & & 0.701 \\
\hline- & 31 & 49.20 & 25 & 43.90 & \\
\hline+ & 26 & 41.30 & 24 & 42.10 & \\
\hline++ & 6 & 9.50 & 8 & 14.00 & \\
\hline Calnexin & & & & & 0.978 \\
\hline- & 34 & 54.00 & 30 & 52.60 & \\
\hline+ & 22 & 34.90 & 20 & 35.10 & \\
\hline++ & 7 & 11.10 & 7 & 12.30 & \\
\hline Calreticulin & & & & & 0.726 \\
\hline- & 41 & 65.10 & 34 & 59.60 & \\
\hline+ & 15 & 23.80 & 14 & 24.60 & \\
\hline++ & 7 & 11.10 & 9 & 15.80 & \\
\hline Tapasin & & & & & 0.832 \\
\hline- & 33 & 52.40 & 27 & 47.40 & \\
\hline+ & 17 & 27.00 & 16 & 28.10 & \\
\hline++ & 13 & 20.60 & 14 & 24.60 & \\
\hline ERAP1 & & & & & 0.119 \\
\hline - & 36 & 57.14 & 30 & 52.63 & \\
\hline+ & 18 & 28.57 & 16 & 28.07 & \\
\hline++ & 9 & 14.29 & 11 & 19.30 & \\
\hline Erp57 & & & & & 0.320 \\
\hline 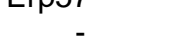 & 27 & 42.86 & 23 & 40.35 & \\
\hline+ & 18 & 28.57 & 16 & 28.07 & \\
\hline++ & 18 & 28.57 & 18 & 31.58 & \\
\hline
\end{tabular}

and facilitating the progress from morphological classification of breast cancer to its molecular classification [5]. Using cDNA microarray technology, some scholars have divided breast cancer into five subtypes. These are ERBB2+type, basal-like type, luminal A type, luminal $B$ type and normal breast-like type.

Based on immunohistochemical features, breast cancer can be divided into HER-2 receptorpositive tumor, hormone receptor-positive tumor, and ER, PR and HER-2 receptor-negative tumors i.e. TNBC [6]. It has been reported that TNBC accounts for about $10-20 \%$ of all breast cancer in China [7]. It accounts for $14.7 \%$ of breast cancer in South Korea, and $15.0 \%$ of breast cancer in Japan [8]. In Europe and America, TNBC constitutes $10-17 \%$ of breast cancer [9]. The disease generally occurs in premenopausal young women, and the prognosis is closely related to disease stage, tumor grade, socio-economic status of the patient, and ethnic nationality [10].

In general, TNBC patients have larger tumor burden and tumor volume than patients with other types of breast cancer, so that their cancers usually have higher malignancy and grade. Moreover, TNBC is characterized by early metastases, short survival time and poor prognosis. The main biological characteristics of TNBC are large neoplasm, infiltrating growth, high lymphatic and distal metastasis rate, hemorrhagic necrosis in the neoplasm, interstitial lymphocyte infiltration, high proportion of grade III tumor, and high mitotic index. 
Table 4: Expression levels of APM member proteins in TNBC patients and non-TNBC patients

\begin{tabular}{|c|c|c|c|c|c|}
\hline \multirow{2}{*}{ Index } & \multicolumn{2}{|c|}{ TNBC $(n=86)$} & \multicolumn{2}{|c|}{ Non-TNBC $(n=34)$} & \multirow{2}{*}{$P$-value } \\
\hline & $\mathbf{N}$ & $\%$ & $\mathbf{n}$ & $\%$ & \\
\hline HLA-A & & & & & 0.170 \\
\hline- & 45 & 52.30 & 21 & 61.80 & \\
\hline+ & 28 & 32.60 & 12 & 35.30 & \\
\hline++ & 13 & 15.10 & 1 & 2.90 & \\
\hline HLA-B & & & & & 0.475 \\
\hline- & 78 & 90.70 & 33 & 97.10 & \\
\hline+ & 7 & 8.10 & 1 & 2.90 & \\
\hline++ & 1 & 1.20 & 0 & 0.00 & \\
\hline HLA-C & & & & & 0.693 \\
\hline- & 82 & 95.30 & 32 & 94.10 & \\
\hline+ & 3 & 3.50 & 2 & 5.90 & \\
\hline++ & 1 & 1.20 & 0 & 0.00 & \\
\hline TAP1 & & & & & 0.072 \\
\hline- & 84 & 97.67 & 33 & 97.06 & \\
\hline+ & 2 & 2.33 & 1 & 2.94 & \\
\hline++ & 0 & 0.00 & 0 & 0.00 & \\
\hline TAP2 & & & & & 0.687 \\
\hline- & 42 & 48.80 & 14 & 41.20 & \\
\hline+ & 35 & 40.70 & 15 & 44.10 & \\
\hline++ & 9 & 10.50 & 5 & 14.70 & \\
\hline Calnexin & & & & & 0.140 \\
\hline- & 41 & 47.70 & 23 & 67.60 & \\
\hline+ & 34 & 39.50 & 8 & 23.50 & \\
\hline++ & 11 & 12.80 & 3 & 8.80 & \\
\hline Calreticulin & & & & & 0.138 \\
\hline- & 49 & 57.00 & 26 & 76.50 & \\
\hline+ & 24 & 27.90 & 5 & 14.70 & \\
\hline++ & 13 & 15.10 & 3 & 8.80 & \\
\hline Tapasin & & & & & 0.003 \\
\hline- & 35 & 40.70 & 25 & 73.50 & \\
\hline+ & 26 & 30.20 & 7 & 20.60 & \\
\hline++ & 25 & 29.10 & 2 & 5.90 & \\
\hline Erp57 & & & & & 0.110 \\
\hline- & 39 & 45.35 & 11 & 32.35 & \\
\hline+ & 24 & 27.91 & 6 & 17.65 & \\
\hline++ & 23 & 26.74 & 13 & 38.24 & \\
\hline ERAP1 & & & & & 0.229 \\
\hline- & 46 & 53.49 & 20 & 58.82 & \\
\hline+ & 24 & 27.91 & 10 & 29.41 & \\
\hline++ & 16 & 18.60 & 4 & 11.76 & \\
\hline
\end{tabular}

Compared with other subtypes of breast cancer, TNBC has a younger age of onset, and is more common in premenopausal women. Moreover, it has a high visceral metastasis rate, high brain metastasis rate, and high rate of local recurrence. In addition, TNBC has a high histological grade, strong invasive ability, high degree of malignancy, late clinical pathological stage, high proliferative and lymph node metastasis rates, and shoved edges. It has been confirmed that the main pathological type of TNBC is ductal carcinoma, which has a high histological grade, and the tumor cells usually express EGFR, P2 cadherin, cytokeratin CK17, CK5/6 and p53, but seldom express E2 cadherin, cyclin D and AR [11].
The immune status of the host is closely connected to the growth, metastasis and diffusion of cancer. The immune system has a dual role in promoting and inhibiting tumor cells during cancer development and progression. Therefore, some scholars put forward the theory of "immune editing", which consists of immune escape, immune surveillance and immune stalemate [12]. Immune escape is considered to be one characteristic of cancer. It has been demonstrated that immune cells that are locally infiltrated by the tumor can convey important therapeutic predictions through inhibiting or advancing the development of breast cancer. Abnormal expression of HLA molecules results in inability to form effective co-stimulatory signals. Thus, tumor cells can escape from the immune surveillance. When an immune recognition 
process is hindered or inhibited, tumors can evade immune surveillance, resulting in promotion of their growth. The activation of immune response demands the co-participation of the antigenic peptide MHC complex recognized by $\mathrm{TCR}$, and the co-stimulatory signals provided by the co-stimulatory ligands and receptors on T cell surface and APC cells. It has been shown that majority of cancer patients have certain immune deficiency characterized by abnormal expression of HLA-I molecules. As a result, HLA-I molecules play an essential role in cancer development and progression [13].

In the present study, results from immunohistochemistry revealed that the positive expressions of HLA-A, HLA-B, TAP2, calnexin, calreticulin, Erp57 and ERAP1 were much lower in breast cancer tissues that those in breast fibroadenoma tissues, while there were no significant differences in the expressions of HLAC, TAP1 and tapasin between benign tissues and breast cancer tissue. These results suggest that the expressions of APM family of genes in breast cancer cells is out of control, a situation which may hinder the correct assembling and loading of HLA-I molecules after transcription, and the presentation of endogenous antigenic peptides.

APM family plays an important role in the assembling and loading of HLA-I molecules. The APM family proteins include TAP, LMP, ERAP1, ERp57, CRT, CNX and tapasin. The proteins TAP1, TAP2, calnexin, calreticulin and tapasin are important molecules involved in antigen processing and presentation. Studies have revealed that down-regulation or deletion of the expression of HLA-I class molecules is important for tumor development. In the process of malignant transformation of cells, the major histocompatibility antigen genes located on chromosome 6 often become very unstable and prone to mutations or chromosomal abnormalities, leading to abnormal expression of HLA-I class antigens [14].

The results of the present study indicate that breast cancer cells inhibit the expression of HLAI class antigens and so block antigen presentation of cancer-specific proteins, thus advancing the cancer survival and development. Therefore, the determination of deletion of the expression of HLA-I antigen has important clinical significance for evaluating prognosis and predicting tumor invasion. The findings of this study reveal no statistical difference in the expression levels of HLA-I members proteins and APM member proteins between Uygur and Han breast cancer patients. This finding suggests that the differences in expressions of HLA-I and APM member proteins between Uyghur and Han breast cancer patients are not significant. It has been reported that compared with Han TNBC patients, Uygur TNBC patients have a lower proportion of $\mathrm{T} 1$ at the time of treatment, but higher proportion of T3 at later stage. However, there was no difference in 5-year tumor-free survival between Han TNBC patients and Uygur TNBC patients. At present, there are limited reports on the clinical pathological features and prognosis of TNBC among various nationalities.

In the current study, there were no statistical differences in the expression levels of HLA-A, HLA-B, HLA-C, TAP1, TAP2, calnexin and calreticulin between TNBC patients and nonTNBC patients. Among the 86 TNBC patients, there were 35 cases of tapasin- $(40.70 \%), 26$ cases of tapasin+ $(30.30 \%)$, and 25 cases of tapasin++ (29.10\%). Among the 34 non-TNBC patients, there were 25 cases of tapasin- $(73.50$ $\%), 7$ cases of tapasin+ $(20.60 \%)$ and 2 cases of tapasin ++ (5.9\%). Thus, the positive expression of tapasin was much higher in TNBC patients than that in non-TNBC patients. Taken together, there were significant differences in the expressions of HLA-I and APM proteins between TNBC patients and non-TNBC patients, manifested in low expressions of HLA-C, TAP1 and calnexin, and high expression of tapasin. Further studies should investigate the molecular mechanisms involved in the expressions of HLA-I and APM proteins in TNBC.

\section{CONCLUSION}

There are no differences in the expressions of HLA-I and APM proteins between Uygur and Han breast cancer patients. However, there are significant differences in the expressions of HLA$I$ and APM proteins between TNBC patients and non-TNBC patients, with low expressions of HLA-C, TAP1 and calnexin, and high tapasin expression. This implies that down-regulation of the expression of HLA-I class of proteins or their deletion is closely related to the prognosis of TNBC. Thus, they can be potential molecular markers in the screening of breast cancer or TNBC.

\section{DECLARATIONS}

\section{Acknowledgement}

This study was supported by Science and Technology Support Program of Xinjiang Uyghur Autonomous Region (no. 201491178). 


\section{Conflict of interest}

No conflict of interest is associated with this work.

\section{Contribution of authors}

We declare that this work was done by the author(s) named in this article and all liabilities pertaining to claims relating to the content of this article will be borne by the authors. All authors read and approved the manuscript for publication. Munire Mushajiang conceived and designed the study. Zhu Xianglu, Gulizhareye Aikula, Mukedaisi Baiketiyaer, Zhang Yujing, Munire Mushajiang collected and analysed the data. Zhu Xianglu wrote the manuscript.

\section{REFERENCES}

1. Dubois S, Mareschal S, Picquenot JM, Viailly PJ, Bohers $E$, Cornic $M$, Bertrand $P$, Veresezan EL, Ruminy $P$, Maingonnat $C$, et al. Immunohistochemical and genomic profiles of diffuse large B-cell lymphomas: Implications for targeted EZH2 inhibitor therapy. Oncotarget 2015; 6(18): 16712-16724.

2. Grange $C$, Tapparo M, Tritta S, Deregibus MS, Battaglia A, Gontero P, Frea B, Camussi G. Role of HLA-G and extracellular vesicles in renal cancer stem cell-induced inhibition of dendritic cell differentiation. BMC Cancer 2015; 15(1): 1009.

3. Jin YP, Valenzuela N, Ziegler M, Rozengurt E, Reed EF. Everolimus Inhibits Anti-HLA I Antibody-Mediated Endothelial Cell Signaling, Migration and Proliferation More Potently Than Sirolimus. Am J Transplant 2014; 14(4): 806-819.

4. World Health Organization. Declaration of Helsinki. Br Med J 1996; 313(7070): 1448-1449.

5. Jucaud V, Ravindranath $\mathrm{MH}$, Terasaki PI. Conformational variants of the individual HLA-I antigens on Luminex single antigen beads used in monitoring HLA antibodies: problems and solutions. Transplantation 2017; 101(4): 764-777.
6. Mehta AM, Spaans VM, Mahendra NB, Osse EM, Vet JN, Purwoto G, Surya IG, Cornian S, Peters AA, Fleuren $G J$, et al. Differences in genetic variation in antigenprocessing machinery components and association with cervical carcinoma risk in two Indonesian populations. Immunogenet 2015; 67(5-6): 267-275.

7. Cui GY. Study on the expression of human leukocyte antigen- $E$ in patients with serous ovarian cancer and its clinical application. Shangdong Univ 2016; 31: 730-737.

8. Das Ghosh D, Mukhopadhyay I, Bhattacharya A, Roy Chowdhury R, Mandal NR, Roy S, Sengupta S. Impact of genetic variations and transcriptional alterations of HLA class I genes on cervical cancer pathogenesis. Int $J$ Cancer 2017; 140(11): 2498-2508.

9. Xue L, Hickling $T$, Song $R$, Nowak J, Rup B. Contribution of enhanced engagement of antigen presentation machinery to the clinical immunogenicity of a human interleukin (IL)-21 receptor-blocking therapeutic antibody. Clin Exp Immunol 2016; 183(1): 102-113.

10. Chang C-C, Pirozzi G, Wen S-H Chung IH, Chiu BL, Errico S, Luongo M, Lombardi ML, Ferrone S. Multiple structural and epigenetic defects in the human leukocyte antigen class I antigen presentation pathway in a recurrent metastatic melanoma following immunotherapy. J Biol Chem 2015; 290(44): 2656226575.

11. Jung JY, Roberts $L L$, Robinson $C M$. The presence of interleukin-27 during monocyte-derived dendritic cell differentiation promotes improved antigen processing and stimulation of $T$ cells. Immunology 2015;144(4): 649-660.

12. Sanin DE, Mountford AP. Sm16, a major component of Schistosoma mansoni cercarial excretory/secretory products, prevents macrophage classical activation and delays antigen processing. Parasit vectors 2015; 8(1): 1.

13. Chen WQ, Ye LJ, Chen FH, Liu J. Study on the value of peripheral HLA-G and serum Th1 and Th2 class cytokines in liver cancer patients. Chin Mod Doctor 2017; 55(14): 1-4.

14. Xu YF, Zhang WL, Hu P, Zhu Y, Fang ZP. Expression of $H L A-F$ in liver cancer cells and its significance in prognosis. Chin J Gen Surg 2014; 29(8): 630-633. 